\title{
Pharmacometabolomics in Drug Discovery \& Development: Applications and Challenges
}

\section{Z. Yang ${ }^{1 *}$ and F. Marotta ${ }^{2}$}

${ }^{1}$ Texas Medical Toxicology, Houston, USA

${ }^{2} R e G e n e r a$ Research Group for Aging Intervention, Milan, Italy

\section{Introduction}

Metabolomics, a relatively new Omics platform, investigate small molecular metabolites by revealing any specific biomarker(s) in human disease or certain metabolism pattern changes after genetic and environmental intervention. It provides a direct read out of the metabolic state of an individual which cannot be obtained from transcriptomics and proteomics [1]. These small molecules are the final products of cellular regulatory processes and may reflect the in vivo metabolism phenotype controlled by both genotype and xenobiotics including: environmental factors, diet and drugs. Metabolomics have been successfully applied in various research and clinical areas including: early diagnosis of diabetes [2], cancers [3], cardiovascular diseases [4], neonatology [5], phytotherapy [6] and even human nutrition research [7].

Recently, the concept of pharmacometabolomics is mentioned more frequently as an emerging discipline to study the effect of drugs on the whole pattern of small endogenous molecules and in applying the profiles of metabolomics for drug development. For the latter part, metabolomics is majorly used to differentiate patients into responder or non-responder groups in an effort to decrease large inter-individual variation in clinical trials $[8,9]$. It is a novel approach that combines metabolite profile and chemo-metrics to model and predict drug targets, efficacy, pharmacokinetics and toxicity on both individual and population basis [8]. It attracts many scientists' attention because of its intrinsic advantages and promising potentials in drug discovery and development [1]. Considering personalized drug treatment is the desired goal for current drug development, pharmacometabolomics provide an effective and inexpensive strategy to evaluate drug efficacy and toxicology, which may make personalized medicine realistic both from scientific and financial perspectives $[10,11]$. Furthermore, the FDA also realized that metabolomics coupling with other "Omics" approaches could be a valuable tool in evaluating general toxicology and could eventually replace the use of animals after addressing certain challenges [12].

Drug discovery and development traditionally relied on various genotype cell/animal models for target identification and validation. With in-silico modeling (i.e., QSAR or ligand-receptor models) in rational drug design, compounds are synthesized and screened for potency and toxicity. Later, time-consuming ADMET (absorption, distribution, metabolism, elimination and toxicity) studies are performed among promising compounds to further screen and identify lead compounds. After optimizing the dosage form and dose regimen of the API, lengthy and costly clinical trials are conducted to provide final evaluation. After several years of development, pharmacometabolomics now provide seamless incorporation to each individual step in drug discovery and development as shown below.

\section{Potential of Diagnosis and Biomarker}

The main application of metabolomics lies in the discovery of biomarkers, which could function as a diagnostic tool for cancer prevention, early detection, and monitoring the progression of diseases $[13,14]$. With high throughput/high resolution mass spectrometry and NMR techniques as well as various statistical models, many biomarkers in human disease were successfully identified to differentiate patients and health control groups, or to predict death [15]. It is very critical for the pharmaceutical industry to find biomarkers for disease and to indicate how people respond to a drug. Biomarkers can be used in predicting therapy response and as surrogate end points for clinical trials [16]. In one study, five metabolites were detected differently between the non-invasive tumors and the invasive tumors in gastric cancer patients, suggesting the selected tissue metabolites could probably be applied for clinical diagnosis of gastric cancer [17]. Many other applications of metabolomics in biomarker discovery have been succinctly summarized in several reviews $[18,19]$.

\section{Drug Target Identification \& Validation}

Since the biochemical bases of many diseases come from the activity of particular enzymes, the pharmacometabolomics profile not only diagnose disease, but also reveal new drug targets and explore new treatment strategies. The alterations of cellular metabolic stages describe combination changes of genome, transcriptome and proteome. Therefore, pharmacometabolomics is a complementary tool for drug target identification and validation. In a serum metabolomic analysis, Chen et al. identified stearoyl-CoA desaturase 1 (SCD1) and its related lipid species which may serve as potential targets for treatment of inflammatory diseases [20]. 37 genetically determined metabolite traits were reported with strong association for various diseases [21]. The identification of associated metabolic traits may generate many new hypotheses for biomedical and pharmaceutical research. Among these genes, SLC16A9 (MCT9) was demonstrated as a carnitine efflux transporter responsible for carnitine efflux from absorptive epithelia into the blood [21]. Wei conducted a target-based metabolomics study to characterize metabolic response of Huh7.5 cells to genomic perturbation of HIF-1. The results identify a new therapeutic target by confirming HIF-1's regulatory role in tumor energy metabolism [22].

\section{ADMET Screening}

ADMET (absorption, disposition, metabolism, elimination and

*Corresponding author: Dr. Zhen Yang, University of Houston, Texas Medical Toxicology, 7707 Fannin Street, Suite 290, Houston, TX, 77054, USA, E-mail: zhen@texasmedtox.com

Received September 18, 2012; Accepted September 18, 2012; Published September 25, 2012

Citation: Yang Z, Marotta F (2012) Pharmacometabolomics in Drug Discovery \& Development: Applications and Challenges. Metabolomics 2:e122. doi:10.4172/2153-0769.1000e122

Copyright: (c) 2012 Yang Z, et al. This is an open-access article distributed under the terms of the Creative Commons Attribution License, which permits unrestricted use, distribution, and reproduction in any medium, provided the original author and source are credited. 
toxicity) could serve as a screen and optimization step in drug discovery and development. The drug candidates with unfavorable ADMET properties (i.e., very poor solubility, extremely low bioavailability, general toxicity) usually failed during the development phase. On the other side, the lead compounds may obtain optimized formulation or dosage design to improve its efficacy and decreased toxicity with good understanding of these biopharmaceutical properties. Drug metabolism is an important activation (for prodrug) or detoxifying route in vivo to access drug's efficacy and toxicity. It determined drug candidate's bioavailability, pharmacokinetics and pharmacodynamics [23]. Coupling with various transporters, drugs including natural compounds may go through extensive metabolism after administration $[24,25]$ and may cause large variations of pharmacokinetics and bioavailability in animal and clinical studies [26,27]. Pharmacometabolomics now provides a faster, simpler, less invasive approach to characterize biopharmaceutical properties and toxicity as well as revealing their mechanisms. In one study, NMR derived pharmacometabolomic analysis showed significant difference of cellular response at high and low doses of docetaxel in MCF7 breast cancer cells [28]. The LC/ MS/MS based pharmacometabolomic approach applied to cisplatin's cytoxotixicty in human lung cancer cell lines was also reported [29]. These results indicated that the efficacy of pharmacometabolomics to evaluate cytotoxicity of various pharmaceutical ingredients and this has been widely accepted [22]. Pharmacometabolomic phenotyping also reveals different responses to xenobiotics in animals. Clayton first demonstrated that a pre-dose metabolic profile of urine could predict the toxicity and metabolism of paracetomol in rats [8]. Two classical experimental models demonstrated that pre-dose urinary metabolic profiles had strong association with post-dose pharmacological outcomes [30].

\section{Applications in Clinical Studies}

As mentioned above, increasing numbers of studies showed practical values of pharmacometabolomics in ADMET and predicted individual drug response and variation. Pharmacometabolomics have been widely applied in clinical studies [31]. Trupp et al. [32] found that metabolite profiling can successfully separate patients into two response groups and could predict LDL-cholesterol response related to simvastatin treatment. Phapale et al. [33] evaluated the potential of a metabolic phenotype to predict individual variation in the pharmacokinetics of tacrolimus. Using four metabolites, one could successfully predict the plasma AUC (area under concentrationtime curve) of tacrolimus in individualized PK. The results indicated that integrative pharmacometabolimic approach combined with the metabolic profiling of pre-dose urine can serve as a useful tool for personalized drug therapy. Clayton showed that $\mathrm{p}$-cresol sulfate reversely correlate to the level of acetaminophen sulfonation in human urine and indicated the metabolism pathway of acetaminophen [34]. These studies demonstrate that monitoring small molecular metabolites in vivo is possible for the development of personalized health care.

\section{Challenges of Pharmacometabolomics}

Although pharmacometabolomics have been successfully applied in drug discovery and development, and are now widely used as complementary tools in understanding biological changes after a drug intervention or a gene knockout, it bears the same challenges as metabolomics faces right now [35]. There are three major challenges ahead in pharmacometabolomics.

Firstly, accurate quantification of small molecular metabolites in cell, tissue, organ or whole organism is the cornerstone of metabolomics. For mass spectrometry based pharmacometabolomics, various analytical aspects including: sample preparation/stability, extraction recovery, carryover and matrix effects, choices of internal standards, and quality control should be systematically validated [36]. For NMR based pharmacometabolomics, the spectrum is generally very complex and difficult to interpret due to function-group based multiple peaks per analyte [22]. Therefore, the quality of the analytical data is very critical for both targeted and non-targeted approaches. We should always keep in mind that compromise and biases do exist when analyzing hundreds of metabolites in various matrix samples without efficient separation, adequate recovery and good reproducibility.

Secondly, selected cohort of samples and sample size should be large enough to confirm pharmacometabolomics results. One example is that there are some controversies about the biomarkers in prostate cancer [37]. The level of sarcosine was reported to show significant elevation in biopsy-positive patients in a high impact journal, indicating its potential to serve as a biomarker for prostate cancer $[38,39]$. However, an independent confirmation in larger cohorts of patients showed negative results for the value of urinary sarcosine as a biomarker for prostate cancer [37]. The underlying reasons may be compounding, but the selection of sample cohorts could significantly contribute to it.

Thirdly, one needs to pay attention to statistical strategies for avoiding false discoveries in metabolomics and related experiments. Many studies failed to take consideration of minimal statistical requirements to obtain true significance by multiple hypothesis testing, inappropriate choice of statistical modeling, and over fitting of experimental data [40]. The classical $p$-values, $p<0.05$, are too optimistic when multiple tests are done simultaneously. There is also a challenge of statistically modeling datasets with large numbers of variables, but relatively small sample sizes [35].

Overall, this editorial is meant to highlight that pharmacometabolomic approach can complement traditional drug discovery, develop processes to identify new pharmacological targets, facilitate drug efficacy and toxicity studies, as well as, reveal new insights into pharmacodynamic and pharmacokinetics. With rapid development of mass spectrometry, NMR techniques and various available statistical methods, pharmacometabolomic will play a more important role through drug discovery and development.

\section{References}

1. Corona G, Rizzolio F, Giordano A, Taffoli G (2011) Pharmaco-metabolomics: an emerging "omics" tool for the personalization of anticancer treatments and identification of new valuable therapeutic targets. J Cell Physiol 227: 2827 2831

2. Suhre K, Meisinger C, Döring A, Altmaier E, Belcredi P, et al. (2010) Metabolic footprint of diabetes: a multiplatform metabolomics study in an epidemiological setting. PLoS One 5: e13953.

3. Trock BJ (2011) Application of metabolomics to prostate cancer. Urol Oncol 29: $572-581$

4. Lewis GD, Asnani A, Gerszten RE (2008) Application of metabolomics to cardiovascular biomarker and pathway discovery. J Am Coll Cardiol 52: 117123.

5. Fanos V, Barberini L, Antonucci R, Atzori L (2012) Pharma-metabolomics in neonatology: is it a dream or a fact?. Curr Pharm Des 18: 2996-3006

6. Yuliana ND, Khatib A, Choi YH, Verpoorte R (2011) Metabolomics for bioactivity assessment of natural products. Phytother Res 25: 157-169.

7. Gibney MJ, Walsh M, Brennan L, Roche HM, German B, et al. (2005) 
Citation: Yang Z, Marotta F (2012) Pharmacometabolomics in Drug Discovery \& Development: Applications and Challenges. Metabolomics 2:e122. doi:10.4172/2153-0769.1000e122

Metabolomics in human nutrition: opportunities and challenges. Am J Clin Nutr 82: 497-503.

8. Clayton TA, Lindon JC, Cloarec O, Antti H, Charuel C, et al. (2006) Pharmacometabonomic phenotyping and personalized drug treatment. Nature 440: 10731077.

9. Lindon JC, Holmes E, Nicholson JK (2006) Metabonomics techniques and applications to pharmaceutical research \& development. Pharm Res 23: 10751088.

10. Malik NN (2012) Biologics: Personalized drugs should cut care costs. Nature 485: 582.

11. Xu EY, Schaefer WH, Xu Q (2009) Metabolomics in pharmaceutical research and development: metabolites, mechanisms and pathways. Curr Opin Drug Discov Devel 12: 40-52.

12. Jacobs A (2009) An FDA perspective on the nonclinical use of the X-Omics technologies and the safety of new drugs. Toxicol Lett 186: 32-35.

13. Denery JR, Nunes AA, Hixon MS, Dickerson TJ, Janda KD (2010) Metabolomics-based discovery of diagnostic biomarkers for onchocerciasis. PLoS Negl Trop Dis 4: e834.

14. Kim YS, Maruvada P, Milner JA (2008) Metabolomics in biomarker discovery: future uses for cancer prevention. Future Oncol 4: 93-102.

15. Herder C, Karakas M, Koenig W (2011) Biomarkers for the prediction of type 2 diabetes and cardiovascular disease. Clin Pharmacol Ther 90: 52-66.

16. Schnackenberg LK, Beger RD (2007) Metabolomic biomarkers: their role in the critical path. Drug Discov Today Technol 4: 13-16.

17. Wu H, Xue R, Tang Z, Deng C, Liu T, et al. (2010) Metabolomic investigation of gastric cancer tissue using gas chromatography/mass spectrometry. Anal Bioanal Chem 396: 1385-1395.

18. Griffiths WJ, Koal T, Wang Y, Kohl M, Enot DP, et al. (2010) Targeted metabolomics for biomarker discovery. Angew Chem Int Ed Engl 49: 54265445.

19. Baumgartner C, OsI M, Netzer M, Baumgertner D, et al. (2011) Bioinformaticdriven search for metabolic biomarkers in disease. J Clin Bioinforma 1: 2.

20. Chen C, Shah YM, Morimura K, Krausz KW, Miyazaki M, et al. (2008) Metabolomics reveals that hepatic stearoyl-CoA desaturase 1 downregulation exacerbates inflammation and acute colitis. Cell Metab 7: 135-147.

21. Suhre K, Shin SY, Petersen AK, Mohney RP, Meredith D, et al. (2011) Human metabolic individuality in biomedical and pharmaceutical research. Nature 477 : 54-60.

22. Wei $R$ (2011) Metabolomics and its practical value in pharmaceutical industry Curr Drug Metab 12: 345-358.

23. Yang Z, Kulkarni K, Zhu W, Hu M, et al. (2012) Bioavailability and Pharmacokinetics of Genistein: Mechanistic Studies on its ADME. Anticancer Agents Med Chem.

24. Yang Z, Zhu W, Gao S, Xu H, Wu B, et al. (2010) Simultaneous determination of genistein and its four phase II metabolites in blood by a sensitive and robust UPLC-MS/MS method: Application to an oral bioavailability study of genistein in mice. J Pharm Biomed Anal 53: 81-90.

25. Yang Z, Zhu W, Gao S, Yin T, Jiang W, et al. (2012) Breast Cancer Resistance Protein (ABCG2) Determines Distribution of Genistein Phase II Metabolites: Reevaluation of the Roles of ABCG2 in the Disposition of Genistein. Drug Metab Dispos 40: 1883-1893.

26. Yang Z, Gao S, Wang J, Yin T, Teng Y, et al. Enhancement of oral bioavailability of $20(\mathrm{~S})$-ginsenoside Rh2 through improved understanding of its absorption and efflux mechanisms. Drug Metab Dispos 39: 1866-1872.

27. Yang Z, Wang JR, Niu T, Gao S, Yin T, et al. (2012) Inhibition of p-glycoprotein leads to improved oral bioavailability of compound $k$, an anticancer metabolite of red ginseng extract produced by gut microflora. Drug Metab Dispos 40 : 1538-1544.

28. Bayet-Robert M, Morvan D, Chollet P, Barthomeuf C (2010) Pharmacometabolomics of docetaxel-treated human MCF7 breast cancer cells provides evidence of varying cellular responses at high and low doses. Breast Cancer Res Treat 120: 613-626.

29. Pyo JS, Ju HK, Park JH, Kwon SW (2008) Determination of volatile biomarkers for apoptosis and necrosis by solid-phase microextraction-gas chromatography/mass spectrometry: a pharmacometabolomic approach to cisplatin's cytotoxicity to human lung cancer cell lines. J Chromatogr B Analyt Technol Biomed Life Sci 876: 170-174.

30. Li H, Ni Y, Su M, Qiu Y, Zhou M, et al. (2007) Pharmacometabonomic phenotyping reveals different responses to xenobiotic in.tervention in rats. $J$ Proteome Res 6: 1364-1370.

31. Nordstrom A, Lewensohn R (2010) Metabolomics: moving to the clinic. J Neuroimmune Pharmacol 5: 4-17.

32. Trupp M, Zhu H, Wikoff WR, Baillie RA, Zeng ZB, et al. (2012) Metabolomics reveals amino acids contribute to variation in response to simvastatin treatment PLoS One 7: e38386.

33. Phapale PB, Kim SD, Lee HW, Lim M, Kale DD, et al. (2010) An integrative approach for identifying a metabolic phenotype predictive of individualized pharmacokinetics of tacrolimus. Clin Pharmacol Ther 87: 426-436.

34. Clayton TA, Baker D, Lindon JC, Everett JR, Nicholson JK (2009) Pharmacometabonomic identification of a significant host-microbiome metabolic interaction affecting human drug metabolism. Proc Natl Acad Sci USA 106: $14728-14733$.

35. Griffin JL, Vidal-Puig A (2008) Current challenges in metabolomics for diabetes research: a vital functional genomic tool or just a ploy for gaining funding? Physiol Genomics 34: 1-5.

36. Koal T, Deigner HP (2010) Challenges in mass spectrometry based targeted metabolomics. Curr Mol Med 10: 216-226.

37. Jentzmik F, Stephan C, Miller K, Schrader M, Erbersdobler A, et al. (2010) Sarcosine in urine after digital rectal examination fails as a marker in prostate cancer detection and identification of aggressive tumours. Eur Urol 58: 12-18.

38. Sreekumar A, Poisson LM, Rajendiran TM, Khan AP, Cao Q, et al. (2010) Sarcosine in urine after digital rectal examination fails as a marker in prostate cancer detection and identification of aggressive tumours. Eur Urol 2010;58:128. Eur Urol 58: e29-30.

39. Sreekumar A, Poisson LM, Rajendiran TM, Khan AP, Cao Q, et al. (2009) Metabolomic profiles delineate potential role for sarcosine in prostate cancer progression. Nature 457: 910-914.

40. Kell DB, Broadhurst DI (2006) Statistical strategies for avoiding false discoveries in metabolomics and related experiments. Metabolomics 2: 171-196. 